\title{
Efficacy and safety of drug-eluting bead transarterial chemoembolization (DEB-TACE) plus apatinib versus DEB-TACE alone in treating huge hepatocellular carcinoma patients
}

\author{
Ningjie $\mathrm{Li}^{1}$ • Jiao Chen ${ }^{2}$ (i)
}

Received: 3 November 2021 / Accepted: 7 December 2021 / Published online: 27 January 2022

(c) The Author(s) 2021

\begin{abstract}
Background Apatinib, a tyrosine kinase inhibitor, inhibits angiogenesis under the tumor hypoxic environment induced by drug-eluting bead transarterial chemoembolization (DEB-TACE), which is hypothesized to have synergic effect with DEBTACE in treating hepatocellular carcinoma (HCC) patients. This study aimed to evaluate the efficacy and safety of DEBTACE plus apatinib in treating huge HCC patients.

Methods Totally, 73 huge HCC patients (tumor size $>10 \mathrm{~cm}$ ) were screened and divided into DEB-TACE plus apatinib group $(N=34)$ or DEB-TACE group $(N=39)$ based on the treatment they received. Their clinical response and adverse events were retrieved. The progression-free survival (PFS) and overall survival (OS) were calculated.

Results DEB-TACE plus apatinib achieved a trend of higher objective response rate (64.7\% vs. $43.6 \%, P=0.071)$, but similar disease control rate $(88.2 \%$ vs. $79.5 \%, P=0.314)$ than DEB-TACE alone. Moreover, DEB-TACE plus apatinib reached an improved PFS (median (95\% CI): 19.0 months (15.5-22.5) vs. 10.9 months $(8.0-13.8), P=0.025)$ and OS (median (95\%CI): 25.1 months (20.3-29.9) vs. 13.7 months (9.8-17.6), $P=0.042)$ than DEB-TACE alone. After adjustment by multivariate Cox's regression analyses, DEB-TACE plus apatinib (vs. DEB-TACE alone) was independently correlated with better PFS (HR: $0.420, P=0.004$ ) and OS (HR: $0.477, P=0.022$ ). Regarding safety, adverse events were mostly mild and manageable; also, they were of no difference between DEB-TACE plus apatinib and DEB-TACE alone (all $P>0.05$ ).

Conclusion DEB-TACE plus apatinib achieves prolonged PFS and OS, while similar adverse events occurrence compared to DEB-TACE alone in huge HCC treatment.
\end{abstract}

Keywords Apatinib · Drug-eluting bead transarterial chemoembolization · Efficacy · Huge hepatocellular carcinoma · Safety

\section{Introduction}

Huge hepatocellular carcinoma (HCC), defined as tumor size above $10 \mathrm{~cm}$ in diameter, places a dramatic disease burden since management options are currently limited, and it usually presents with unfavorable survival [1-6]. For instance, most huge HCC patients experience tumor invasion and

Jiao Chen

jiaosi738591669@163.com

1 Department of Radiology, Wuhan Sixth Hospital, Affiliated Hospital of Jianghan University, Wuhan 430015, China

2 Department of Radiology, Edong Healthcare Group, Huangshi Central Hospital, Affiliated Hospital of Hubei Polytechnic University, No.141 Tianjin Raod, Huangshi 435000, China intrahepatic metastasis, which misses the opportunity of surgical resection as the curative treatment $[2,5,6]$. Consequently, transarterial chemoembolization (TACE) is commonly applied in treating huge HCC patients [7-9]. Despite displaying certain efficacy and safety, TACE may cause disease progression and result in an unfavorable survival profile in huge HCC patients due to neoangiogenesis arising from the hypoxic environment of tumor blood vessels [8-10]. Hence, developing novel therapies to improve the prognosis of huge HCC patients is urgent and necessary.

Apatinib, a tyrosine kinase inhibitor selectively targeting vascular endothelial growth factor receptor-2 (VEGFR2), may synergize with TACE in treating HCC patients via inhibiting angiogenesis in the tumor hypoxic environment caused by TACE [10-13]. For instance, TACE plus apatinib achieves better short-term and long-term treatment efficacy 
as well as similar incidence of adverse events compared to TACE alone in treating advanced HCC patients, recurrent HCC patients, and huge HCC patients [14-18]. Drug-eluting bead TACE (DEB-TACE), as an improved and innovated type of TACE, utilizes non-absorbable microspheres as embolic agent and drug-loaded material to the target tumor region in treating HCC patients [19]. Moreover, DEB-TACE exhibits several advantages over conventional TACE (such as improved embolic effect, less safety issue, better loading and releasing profile etc.) [19]. Few studies apply DEBTACE plus apatinib in treating huge HCC patients.

Thus, this study aimed to compare the efficacy and safety of DEB-TACE plus apatinib versus DEB-TACE alone in huge HCC patients.

\section{Methods}

\section{Subjects}

This study screened a total of 73 huge HCC patients who were treated by DEB-TACE or DEB-TACE plus apatinib from January 2018 to December 2020. The screening criteria were set as (i) diagnosed as HCC, (ii) largest tumor size $\geq 10 \mathrm{~cm}$, (iii) at least one treatment response data was available, (iv) clinical data before operation were accessible, and (v) treated by DEB-TACE or DEB-TACE plus apatinib. The patients with other primary cancers or hematological malignancies at diagnosis were excluded from the study. After screening, 73 huge HCC patients were divided into two groups according to the different treatment regimens: DEB-TACE group, 39 patients with DEB-TACE therapy and DEB-TACE plus apatinib group, 34 patients with DEBTACE plus apatinib therapy. The study was approved by the Institutional Review Board.

\section{Data collection}

Patients' characteristics were collected from medical records for the further analysis, including demographic information, disease information, tumor marker, liver function indexes, treatment history, and cycles of DEB-TACE.

\section{Treatment procedure}

All patients in the study underwent DEB-TACE therapy, and the procedure was the same as a previous study [20]. Briefly, femoral artery was pierced by Seldinger technique, and tumor-supplying artery was identified by hepatic angiography; then the tumor-feeding artery was catheterized with microcatheters by super-selective catheterization, following that microspheres (CalliSpheres bead, HepaSpheres bead or DC bead) were applied as carriers to load pirarubicin and were slowly injected into the tumor-supplying artery. The embolization was completed when staining of tumor was disappeared. For DEB-TACE plus apatinib group, 1 week after DEB-TACE operation, apatinib was taken orally at a dose of $500 \mathrm{mg}$ daily, and discontinued 1 week before the next DEB-TACE operation. After the last DEB-TACE operation, patients received apatinib continually at a dose of $500 \mathrm{mg}$ daily, and the use of apatinib was continued until progression of tumor, intolerance of patients, or death of patients. Intolerance of patients was defined as patients who experienced adverse events and required dose adjustment or discontinuation when receiving apatinib. For the patients who had serious adverse events while receiving apatinib, symptomatic treatment was performed, and the dose of apatinib was halved. If there was no remission of adverse events after symptomatic treatment, apatinib was discontinued.

\section{Assessment}

Clinical responses (including complete response (CR), partial response (PR), stable disease (SD), and progressive disease (PD)) were evaluated in accordance with the modified Response Evaluation Criteria in Solid Tumors (mRECIST) [21], and the data were obtained from the clinical records, which were assessed 6-10 weeks after initiation of first DEB-TACE operation. Then, objective response rate (ORR) and disease-control rate (DCR) were calculated furtherly. Besides, adverse events and liver function indexes (alanine aminotransferase (ALT) and aspartate aminotransferase (AST)) at 6-10 weeks after initiation of first DEBTACE operation were retrieved for safety assessment of the treatment. In addition, follow-up data of all patients were abstracted from the medical records as well. The final date of follow-up was August 31, 2021, based on which progression-free survival (PFS) and overall survival (OS) were calculated for prognostic analysis.

\section{Statistical analysis}

SPSS 21.0 statistical software (IBM Corp., Armonk, New York, USA) and GraphPad Prism 7.02 (GraphPad Software Inc., San Diego, California, USA) were used for analysis and plotting, respectively. Patients' characteristics, clinical responses, and adverse events between groups were compared using Student's $t$ test, Wilcoxon rank sum test, chisquare test, and Fisher's exact test. PFS and OS were elucidated using Kaplan-Meier (KM) curves, and the median PFS and OS with 95\% confidence interval (CI) were calculated as well. The KM curves were analyzed by log-rank test and Gehan-Breslow-Wilcoxon test. Factors affecting PFS and OS were determined using multivariable Cox's proportional hazard regression model analyses with forward stepwise (conditional) method, and all patient's characteristics 
were included in the analysis. A $P$ value $<0.05$ indicated statistical significance.

\section{Results}

\section{Huge HCC patients' clinical features}

The mean ages of patients in DEB-TACE plus apatinib group and DEB-TACE group were $58.3 \pm 8.5$ years and $60.4 \pm 8.5$ years, respectively (Table 1 ). Moreover, there were $6(17.6 \%)$ females and $28(82.4 \%)$ males in the DEBTACE plus apatinib group then $3(7.7 \%)$ females and 36 (92.3\%) males in DEB-TACE group. Moreover, 5 (14.7\%) and $2(5.1 \%)$ patients had history of tumorectomy in DEBTACE plus apatinib group and DEB-TACE group, respectively. By comparison, there was no difference of clinical features between DEB-TACE plus apatinib group and DEB-TACE group including demographic information, disease information, tumor marker, treatment history, and cycles of current DEB-TACE (all $P>0.05$ ). The detailed clinical features of huge HCC patients are listed in Table 1.

Table 1 Patients' characteristics

\begin{tabular}{|c|c|c|c|}
\hline Items & DEB-TACE group $(N=39)$ & $\begin{array}{l}\text { DEB-TACE plus } \\
\text { apatinib group } \\
(N=34)\end{array}$ & $P$ value \\
\hline \multicolumn{4}{|l|}{ Demographic information } \\
\hline Age (years), mean $\pm \mathrm{SD}$ & $60.4 \pm 8.5$ & $58.3 \pm 8.5$ & 0.304 \\
\hline Gender, no. (\%) & & & 0.288 \\
\hline Female & $3(7.7)$ & $6(17.6)$ & \\
\hline Male & $36(92.3)$ & $28(82.4)$ & \\
\hline History of alcohol, no. (\%) & $11(28.2)$ & $9(26.5)$ & 0.868 \\
\hline History of HBV, no. (\%) & $28(71.8)$ & $22(64.7)$ & 0.515 \\
\hline History of liver cirrhosis, no. (\%) & $21(53.8)$ & $13(38.2)$ & 0.182 \\
\hline \multicolumn{4}{|l|}{ Disease information } \\
\hline ECOG PS score, no. (\%) & & & 0.663 \\
\hline 0 & $26(66.7)$ & $21(61.8)$ & \\
\hline 1 & $13(33.3)$ & $13(38.2)$ & \\
\hline Child-Pugh class, No. (\%) & & & 0.964 \\
\hline A & $22(56.4)$ & $19(55.9)$ & \\
\hline $\mathrm{B}$ & $17(43.6)$ & $15(44.1)$ & \\
\hline Largest tumor size $(\mathrm{cm})$, median (IQR) & $11.8(10.5-12.7)$ & $11.0(10.1-13.3)$ & 0.300 \\
\hline Portal vein invasion, No. (\%) & $11(28.2)$ & $14(41.2)$ & 0.244 \\
\hline Hepatic vein invasion, No. (\%) & $6(15.4)$ & $6(17.6)$ & 0.795 \\
\hline BCLC stage, No. (\%) & & & 0.129 \\
\hline B & $23(59.0)$ & $14(41.2)$ & \\
\hline $\mathrm{C}$ & $16(41.0)$ & $20(58.8)$ & \\
\hline \multicolumn{4}{|l|}{ Tumor marker } \\
\hline AFP $(\mu \mathrm{g} / \mathrm{L})$, median $(\mathrm{IQR})$ & $165.4(18.9-2556.6)$ & $483.1(27.8-6438.8)$ & 0.257 \\
\hline \multicolumn{4}{|l|}{ Treatment history } \\
\hline History of tumorectomy, No. (\%) & $2(5.1)$ & $5(14.7)$ & 0.240 \\
\hline History of ablation, No. (\%) & $4(10.3)$ & $4(11.8)$ & 1.000 \\
\hline History of TACE, No. (\%) & $10(25.6)$ & $13(38.2)$ & 0.248 \\
\hline Cycles of current DEB-TACE & & & 0.730 \\
\hline 1 cycle, no. $(\%)$ & $23(59.0)$ & $18(52.9)$ & \\
\hline 2 cycles, no. $(\%)$ & $8(20.5)$ & $10(29.4)$ & \\
\hline 3 cycles, no. $(\%)$ & $8(20.5)$ & $5(14.7)$ & \\
\hline 4 cycles, no. $(\%)$ & $0(0.0)$ & $1(2.9)$ & \\
\hline
\end{tabular}

$D E B-T A C E$ drug-eluting bead transarterial chemoembolization, $S D$ standard deviation, $H B V$ hepatitis B virus, ECOG PS Eastern Cooperative Oncology Group performance status, IQR interquartile range, $B C L C$ Barcelona Clinic Liver Cancer, $A F P$ alpha-fetoprotein, TACE transarterial chemoembolization 
Table 2 Clinical responses

\begin{tabular}{lllc}
\hline Items & $\begin{array}{l}\text { DEB-TACE group } \\
(N=39)\end{array}$ & $\begin{array}{l}\text { DEB-TACE plus apatinib } \\
\text { group }(N=34)\end{array}$ & $P$ value \\
\hline $\begin{array}{l}\text { Clinical response, no. }(\%) \\
\text { CR }\end{array}$ & $0(0.0)$ & $1(2.9)$ & 0.064 \\
PR & $17(43.6)$ & $21(61.8)$ & \\
SD & $14(35.9)$ & $8(23.5)$ & \\
PD & $8(20.5)$ & $4(11.8)$ & 0.071 \\
ORR (CR + PR), no. $(\%)$ & $17(43.6)$ & $22(64.7)$ & 0.314 \\
DCR (CR + PR+SD), no. $(\%)$ & $31(79.5)$ & $30(88.2)$ & \\
\hline
\end{tabular}

$D E B-T A C E$ drug-eluting bead transarterial chemoembolization, $C R$ complete response, $P R$ partial response, $S D$ stable disease, $P D$ progressive disease, $O R R$ objective response rate, $D C R$ disease control rate

\section{Comparison of clinical response}

In DEB-TACE plus apatinib group, there were $1(2.9 \%), 21$ (61.8\%), 8 (23.5\%), and 4 (11.8\%) patients who reached CR, $\mathrm{PR}, \mathrm{SD}$, and PD, respectively, while in DEB-TACE group, 0 (0.0\%), 17 (43.6\%), 14 (35.9\%), and 8 (20.5\%) patients achieved CR, PR, SD, and PD, respectively (Table 2). By comparison, DEB-TACE plus apatinib group exhibited a trend of higher ORR $(64.7 \%$ vs. $43.6 \%, P=0.071)$, but similar DCR (88.2\% vs. $79.5 \%, P=0.314$ ) as DEB-TACE group.

\section{Comparison of survival profile}

The PFS was prolonged in DEB-TACE plus apatinib group compared to DEB-TACE group (median (95\% CI): 19.0 months (15.5-22.5) vs. 10.9 months (8.0-13.8)) $(P=0.025$ : Fig. 1A). Moreover, the OS was also longer in DEB-TACE plus apatinib group than DEB-TACE group (median (95\%CI): 25.1 months (20.3-29.9) vs. 13.7 months (9.8-17.6)), $(P=0.042$ : Fig. 1B).

Further subgroup analysis displayed that PFS $(P=0.059)$ was similar, while OS $(P=0.037)$ was longer in DEB-TACE group plus apatinib group compared to DEB-TACE group in huge HCC patients with history
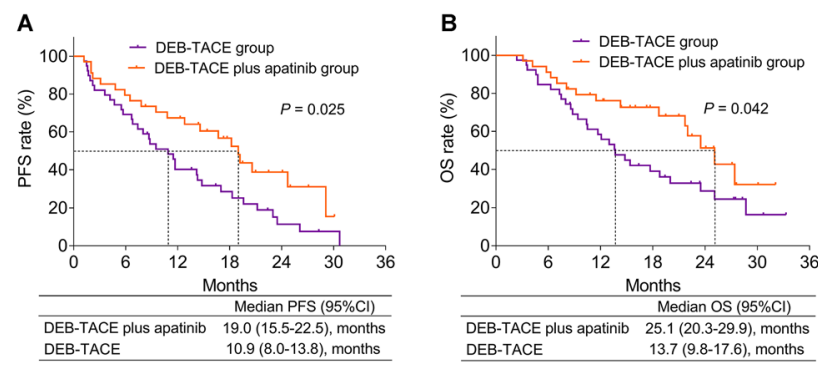

Fig. 1 DEB-TACE plus apatinib achieved improved prognosis compared to DEB-TACE alone. Comparison of PFS (A) and OS (B) between huge HCC patients received DEB-TACE plus apatinib and DEB-TACE alone therapies of TACE (Supplementary Fig. 1A, B). Besides, PFS $(P=0.036)$ and $\mathrm{OS}(P=0.049)$ were also prolonged in DEB-TACE plus apatinib group than DEB-TACE group in huge HCC patients without history of TACE (Supplementary Fig. 1C, D).

\section{Adjustment by multivariate Cox's analyses}

After adjustment by multivariate Cox's regression model, DEB-TACE plus apatinib (vs. DEB-TACE) was independently correlated with improved PFS (hazard ratio (HR): $0.420,95 \%$ CI: $0.232-0.761, P=0.004$, Fig. $2 A)$ and OS (HR: $0.477,95 \%$ CI: $0.253-0.898, P=0.022$ : Fig. $2 \mathrm{~B}$ ). Moreover, portal vein invasion (yes vs. no) (HR: 2.639, 95\% CI: $1.492-4.667, P=0.001)$ and history of tumorectomy (yes vs. no) (HR: 2.928, 95\%CI: 1.147-7.474, $P=0.025)$ were independently associated with shorter PFS. Furthermore, portal vein invasion (yes vs. no) served as an independent factor for reduced OS (HR: 3.124, 95\%CI: $1.704-5.727, P<0.001)$.

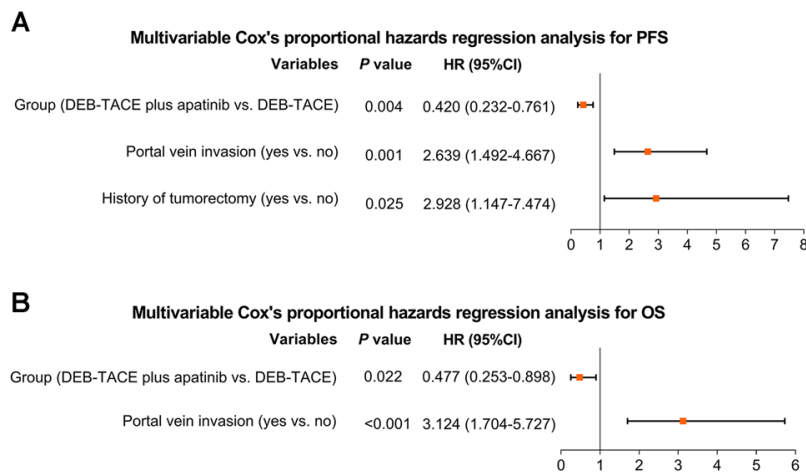

Fig. 2 DEB-TACE plus apatinib (vs. DEB-TACE) independently correlated with prolonged PFS and OS. Multivariate Cox's proportional hazards regression analyses for PFS (A) and OS (B) in huge HCC patients 


\section{Comparison of adverse events}

The commonly observed adverse events in DEB-TACE plus apatinib group were pain (50.0\%), fever (26.5\%), hand-footskin reaction (26.5\%), nausea/vomiting (17.6\%), blood pressure elevation (11.8\%), diarrhea (5.9\%) etc. (Table 3). Meanwhile, in DEB-TACE group, pain (46.2\%), fever (17.9\%), nausea/vomiting (15.4\%), hand-foot-skin reaction (10.3\%), diarrhea (10.3\%), blood pressure elevation (5.1\%) etc. were frequently reported. By comparison, these above-mentioned adverse events were of no difference between DEB-TACE plus apatinib group and DEB-TACE group (all $P>0.05$ ).

\section{Change of liver function indexes}

ALT level was similar before therapy $(P=0.404)$, while it was higher after therapy in DEB-TACE plus apatinib group than DEB-TACE group $(P=0.036)$ (Fig. 3A). However, there was no difference of AST level between DEB-TACE plus apatinib group and DEB-TACE group before therapy $(P=0.362)$ or after therapy $(P=0.114)$ (Fig. 3B).

\section{Discussion}

DEB-TACE is developed as an improved technique over conventional TACE since it exhibits several advantages: (a) DEB-TACE enables a controlled manner of releasing loaded drug, (b) DEB-TACE increases intratumoral concentration and achieves a better anti-tumor effect, (c) DEB-TACE realizes an improved embolic effect by using non-soluble microspheres, (d) DEB-TACE is more convenient and feasible in the clinical practice, (e) the drug-loading speed and amount of DEB-TACE is better, and (f) DEB-TACE preciously targets HCC tumor tissue and reduces the systematic cytotoxicity [19]. However, due to enhanced tumor angiogenesis from hypoxic tumor environment caused by DEB-TACE, increased risk of disease progression and tumor recurrence in HCC patients has been reported $[10,22]$. The emergence of apatinib suppresses this DEB-TACE-induced angiogenetic process
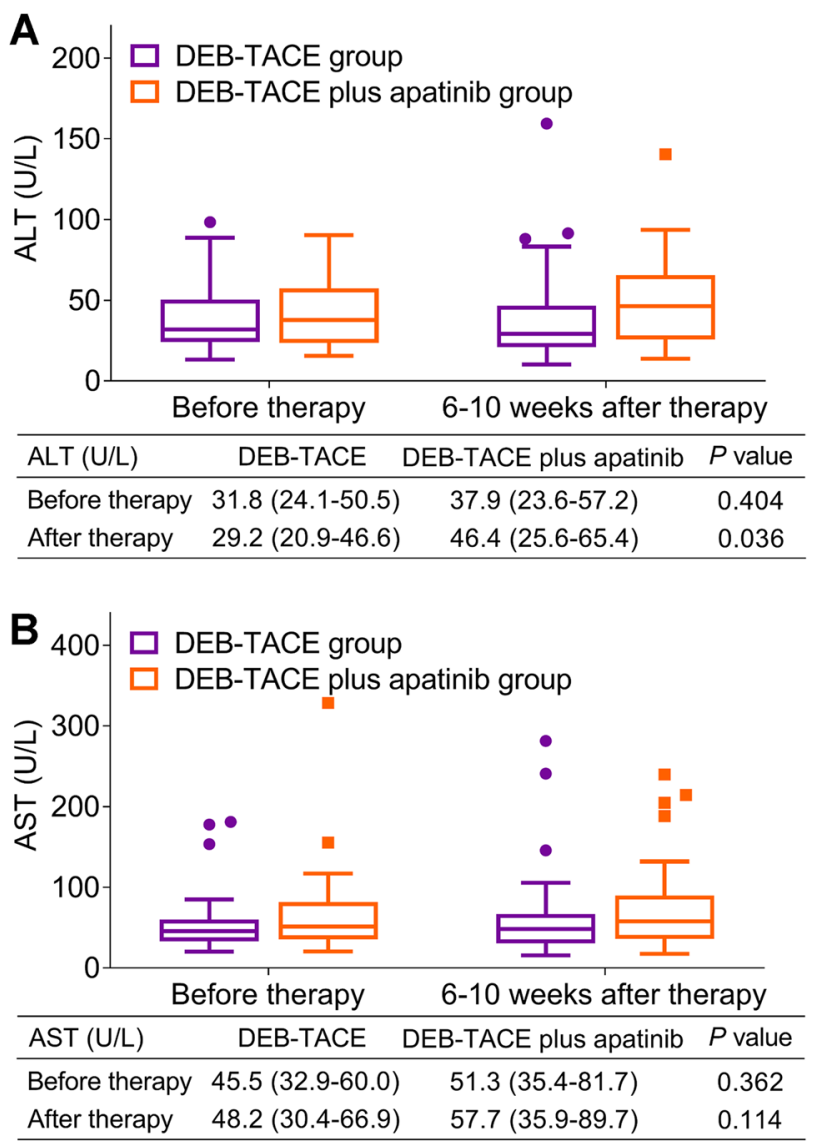

Fig. 3 DEB-TACE plus apatinib induced a slight increase of ALT level than DEB-TACE alone. Comparison of ALT level in huge HCC patients between DEB-TACE plus apatinib group and DEB-TACE group before and after therapy (A). Comparison of AST level in huge HCC patients between DEB-TACE plus apatinib group and DEBTACE group before and after therapy $(\mathbf{B})$

arising from hypoxia tumor environment, implying its ability to synergize with DEB-TACE in HCC patients. No study compares the treatment response of DEB-TACE plus apatinib with DEB-TACE alone in huge HCC patients. In the current study, we observed that DEB-TACE plus apatinib achieved a trend of higher ORR and clinical response rate but similar DCR as DEB-TACE alone in huge HCC

\begin{tabular}{llll}
\hline Items & $\begin{array}{l}\text { DEB-TACE group } \\
(N=39)\end{array}$ & $\begin{array}{l}\text { DEB-TACE plus apatinib } \\
\text { group }(N=34)\end{array}$ & $P$ value \\
\hline Pain, no. (\%) & $18(46.2)$ & $17(50.0)$ & 0.743 \\
Fever, no. (\%) & $7(17.9)$ & $9(26.5)$ & 0.380 \\
Nausea/vomiting, no. (\%) & $6(15.4)$ & $6(17.6)$ & 0.795 \\
Hand-foot-skin reaction, no. (\%) & $4(10.3)$ & $9(26.5)$ & 0.071 \\
Diarrhea, no. (\%) & $4(10.3)$ & $2(5.9)$ & 0.679 \\
Blood pressure elevation, no. $(\%)$ & $2(5.1)$ & $4(11.8)$ & 0.408 \\
\hline
\end{tabular}

$D E B$-TACE drug-eluting bead transarterial chemoembolization 
patients, which could be explained as that (a) the sample size of the current study was limited, leading to a low statistical power; thus, these results were not statistically significant. (b) The obvious effect of apatinib on inhibiting angiogenesis might require an accumulating period of time, which implied that 6-10-week duration was relatively short for its treatment response evaluation.

Previous study illustrates that TACE plus apatinib realizes an increased OS than TACE alone, also TACE plus apatinib (vs. TACE alone) is independently correlated with longer OS in HCC patients with macroscopic vascular invasion [14]. Moreover, several studies also exhibit that TACE plus apatinib achieves a favorable survival profile compared to TACE alone in treating advanced unresectable HCC patients, while none of them applies DEB-TACE for therapy [15-18]. Regarding DEB-TACE plus apatinib in intermediate-to-advanced HCC patients (BCLC stage B/C), DEB-TACE plus apatinib achieves a PFS of 9.5 months (95\% CI: 8.1-10.9 months) and OS of 22.0 months (95\% CI: 20.2-23.9 months), but it is a single-arm study which lacks a control group [23]. In the present study, we revealed that DEB-TACE plus apatinib exhibited a median PFS of 19.0 months (95\% CI: 15.5-22.5 months) and median OS of 25.1 months (95\%CI: 20.3-29.9 months), which were obviously favorable than DEB-TACE alone therapy in treating huge HCC patients. Also, DEB-TACE plus apatinib (vs. DEB-TACE) could independently correlate with prolonged PFS and OS, which could be explained as that (a) apatinib inhibited the angiogenesis caused by DEB-TACE, and thereby led to a synergetic effect of DEB-TACE plus apatinib in huge HCC patients [24]. Thus, DEB-TACE plus apatinib might reduce risk of disease progression and recurrence, and thereby resulted in a favorable prognosis. (b) DEB-TACE plus apatinib exhibited a numerical higher ORR than DEBTACE alone and thus led to an improved survival profile in huge HCC patients.

Apart from the efficacy, the safety issue of TACE plus apatinib is of great attention in treating HCC patients as well. For instance, TACE plus apatinib induces a similar incidence rate of adverse events as TACE alone in advanced HCC patients (including fever, abdominal pain, nausea/vomiting, hypertension, hand-foot syndrome, diarrhea etc.) [16]. Regarding the safety of DEB-TACE plus apatinib, it induces moderate and manageable adverse events in advanced HCC patients; also, it does not influence liver function indexes [23]. In the present study, we observed that DEB-TACE plus apatinib showed a similar incidence rate of adverse events as DEB-TACE alone in huge HCC patients. ALT level was slightly elevated after therapy in huge HCC patients received DEB-TACE plus apatinib compared to those received DEBTACE alone, which could be explained as that apatinib selectively inhibited cytochrome P450 enzyme (such as CYP2B6/2B1 and CYP2D6/2D1), which might lead to an impaired liver function and slight elevation of ALT level in huge HCC patients [25].

There were some limitations in the current study. For example, the sample size of the current study was relatively small; also, this study was a retrospective study, which might cause patient selection bias; thus, further prospective study with larger sample size to validate our findings in huge HCC patients was necessary. Moreover, apatinibinduced adverse events (such as abnormal coagulation indexes, proteinuria etc.) and the grade of adverse events were not recorded in the present study; therefore, further study could focus on this point. Furthermore, the treatment option after disease progression was not recorded since most huge HCC patients did not live in the local region requiring long-distant travelling to our hospital.

In conclusion, DEB-TACE plus apatinib achieves prolonged PFS and OS, while similar adverse event occurrence compared to DEB-TACE alone in huge HCC treatment.

Supplementary information The online version contains supplementary material available at https://doi.org/10.1007/s11845-021-02884-w.

\section{Declarations}

Ethics approval The study was approved by the Institutional Review Board.

Conflict of interest The authors declare no competing interests.

Open Access This article is licensed under a Creative Commons Attribution 4.0 International License, which permits use, sharing, adaptation, distribution and reproduction in any medium or format, as long as you give appropriate credit to the original author(s) and the source, provide a link to the Creative Commons licence, and indicate if changes were made. The images or other third party material in this article are included in the article's Creative Commons licence, unless indicated otherwise in a credit line to the material. If material is not included in the article's Creative Commons licence and your intended use is not permitted by statutory regulation or exceeds the permitted use, you will need to obtain permission directly from the copyright holder. To view a copy of this licence, visit http://creativecommons.org/licenses/by/4.0/.

\section{References}

1. Yin Y, Cheng JW, Chen FY et al (2021) A novel preoperative predictive model of 90-day mortality after liver resection for huge hepatocellular carcinoma. Ann Transl Med 9(9):774. https://doi. org/10.21037/atm-20-7842

2. Lim C, Compagnon P, Sebagh M et al (2015) Hepatectomy for hepatocellular carcinoma larger than $10 \mathrm{~cm}$ : preoperative risk stratification to prevent futile surgery. HPB (Oxford) 17(7):611623. https://doi.org/10.1111/hpb.12416

3. Yu SCH, Hui JW, Li L et al (2021) Comparison of chemoembolization, radioembolization, and transarterial ethanol ablation for huge hepatocellular carcinoma $(>/=10 \mathrm{~cm})$ in tumour response and long-term survival outcome. Cardiovasc Intervent Radiol. https://doi.org/10.1007/s00270-021-02777-6 
4. Truty MJ, Vauthey JN (2010) Surgical resection of high-risk hepatocellular carcinoma: patient selection, preoperative considerations, and operative technique. Ann Surg Oncol 17(5):1219-1225. https://doi.org/10.1245/s10434-010-0976-5

5. Bhanu JS, Venkitaraman B, Palaniappan R et al (2020) Prognostic factors and survival outcomes of surgical resection of huge hepatocellular carcinomas. J Gastrointest Cancer 51(1):250-253. https:// doi.org/10.1007/s12029-019-00240-x

6. Tsoulfas G, Mekras A, Agorastou P et al (2012) Surgical treatment for large hepatocellular carcinoma: does size matter? ANZ J Surg 82(7-8):510-517. https://doi.org/10.1111/j.1445-2197.2012.06079.x

7. Chang Y, Jeong SW, Young Jang J et al (2020) Recent updates of transarterial chemoembolilzation in hepatocellular carcinoma. Int J Mol Sci 21(21):8165. https://doi.org/10.3390/ijms21218165

8. Xue T, Le F, Chen R et al (2015) Transarterial chemoembolization for huge hepatocellular carcinoma with diameter over ten centimeters: a large cohort study. Med Oncol 32(3):64. https:// doi.org/10.1007/s12032-015-0504-3

9. Miyayama S, Kikuchi Y, Yoshida M et al (2019) Outcomes of conventional transarterial chemoembolization for hepatocellular carcinoma $>/=10 \mathrm{~cm}$. Hepatol Res 49(7):787-798. https://doi.org/ 10.1111/hepr.13335

10. Petrillo M, Patella F, Pesapane F et al (2018) Hypoxia and tumor angiogenesis in the era of hepatocellular carcinoma transarterial loco-regional treatments. Future Oncol 14(28):2957-2967. https:// doi.org/10.2217/fon-2017-0739

11. Scott LJ (2018) Apatinib: a review in advanced gastric cancer and other advanced cancers. Drugs 78(7):747-758. https://doi.org/10. 1007/s40265-018-0903-9

12. Liu K, Zhang X, Xu W et al (2017) Targeting the vasculature in hepatocellular carcinoma treatment: starving versus normalizing blood supply. Clin Transl Gastroenterol 8(6):e98. https://doi.org/ 10.1038/ctg.2017.28

13. Wang Z, Zhou W, Zhang H et al (2015) Combination of antiangiogenesis agents and transarterial embolization: is it a promising approach for the treatment of liver cancer? Discov Med 20(108):51-55

14. Shen L, Chen S, Qiu Z et al (2020) Transarterial chemoembolization combined with apatinib versus transarterial chemoembolization alone for hepatocellular carcinoma with macroscopic vascular invasion: a propensity score matching analysis. J Cancer Res Ther 16(5):1063-1068. https://doi.org/10.4103/jcrt.JCRT_801_19

15. Chen S, Yu W, Zhang K et al (2018) Comparison of the efficacy and safety of transarterial chemoembolization with and without apatinib for the treatment of BCLC stage $\mathrm{C}$ hepatocellular carcinoma. BMC Cancer 18(1):1131. https://doi.org/10.1186/s12885-018-5081-3
16. Zhu Y, Feng B, Mei L et al (2019) Clinical efficacy of TACE combined with apatinib in the treatment of advanced hepatocellular carcinoma. J BUON 24(2):608-614

17. Gu H, Li J, You N et al (2020) Efficacy and safety of apatinib combined with transarterial chemoembolization (TACE) in treating patients with recurrent hepatocellular carcinoma. Ann Transl Med 8(24):1677. https://doi.org/10.21037/atm-20-7244

18. Liu J, Xie S, Duan X et al (2020) Assessment of efficacy and safety of the transcatheter arterial chemoembolization with or without apatinib in the treatment of large hepatocellular carcinoma. Cancer Chemother Pharmacol 85(1):69-76. https://doi.org/ 10.1007/s00280-019-04004-Z

19. Melchiorre F, Patella F, Pescatori L et al (2018) DEB-TACE: a standard review. Future Oncol 14(28):2969-2984. https://doi.org/ 10.2217/fon-2018-0136

20. Liu Y, Huang W, He M et al (2019) Efficacy and safety of CalliSpheres $(\mathrm{R}))$ drug-eluting beads transarterial chemoembolization in Barcelona clinic liver cancer stage C patients. Oncol Res 27(5):565573. https://doi.org/10.3727/096504018X15313896322888

21. Lencioni R, Llovet JM (2010) Modified RECIST (mRECIST) assessment for hepatocellular carcinoma. Semin Liver Dis 30(1):52-60. https://doi.org/10.1055/s-0030-1247132

22. Sergio A, Cristofori C, Cardin R et al (2008) Transcatheter arterial chemoembolization (TACE) in hepatocellular carcinoma (HCC): the role of angiogenesis and invasiveness. Am J Gastroenterol 103(4):914-921. https://doi.org/10.1111/j.1572-0241.2007.01712.x

23. Liu J, Xu J, Zhang W et al (2020) Safety and efficacy of drugeluting bead transarterial chemoembolization combined with apatinib in patients with advanced hepatocellular carcinoma. Acad Radiol 27(5):704-709. https://doi.org/10.1016/j.acra.2019. 07.003

24. Yang C, Qin S (2018) Apatinib targets both tumor and endothelial cells in hepatocellular carcinoma. Cancer Med 7(9):4570-4583. https://doi.org/10.1002/cam4.1664

25. Bao SS, Wen J, Zheng X et al (2018) Evaluation of the inhibition effects of apatinib on human and rat cytochrome P450. Toxicol Lett 297:1-7. https://doi.org/10.1016/j.toxlet.2018.08.010

Publisher's Note Springer Nature remains neutral with regard to jurisdictional claims in published maps and institutional affiliations. 